ture equivalent, $10 \mathrm{~K}$. Although this is a relatively small energy scale, it makes its presence felt through the strong magnetoelectronic coupling - a flip of a spin in an applied magnetic field can change the local electronic structure from insulating to metallic, so affecting the energy balance on a (larger) electronic scale.

Another potentially important contributor to the properties of the manganites is a Jahn-Teller structural instability; the lattice surrounding the $\mathrm{Mn}^{3+}$ ions distorts to lift a degeneracy in the energy levels of the electronic ground state of these ions, and so lowers the energy of the entire (electronic+lattice) system. The electronic configuration of the $\mathrm{Mn}^{3+}$ ions is $d^{4}$ with all spins aligned, with all three $t_{2 g}$ orbitals fully occupied, but only single occupation of the two degenerate $e_{g}$ orbitals (which point directly at the neighbouring oxygen ions).

The ideal structure of these compounds is simple cubic perovskite. But the parent compound of the CMR materials, $\mathrm{LaMnO}_{3}$, has a complex structure (see figure), which can be described as a series of distortions from the simple cubic structure: rotation of the $\mathrm{MnO}_{6}$ octahedra around the $z$ axis; tilting of the octahedra about an axis in the $x-y$ plane; distortion of $\mathrm{MnO}_{6}$ octahedra resulting in three unequal $\mathrm{Mn}-\mathrm{O}$ bond lengths; and relaxation of the entire structure to give three unequal lattice constants. The rotational motions result from a misfit of ionic sizes whereas the distortion of the $\mathrm{MnO}_{6}$ octahedron is widely believed to be the manifestation of the Jahn-Teller instability. As $\mathrm{Ca}$ is substituted for $\mathrm{La}$, the distortion of the $\mathrm{MnO}_{6}$ octahedra nearly vanishes according to diffraction experiments that measure the average structure. But there has been speculation that the Jahn-Teller distortion nevertheless remains, either as an incoherent local distortion (fluctuations in space) or, more exotically, as dynamical Jahn-Teller fluctuations.

Although invoking the Jahn-Teller mechanism to account for data has been common, all phenomena up to this point could in principle be addressed in terms of static atoms. The new result of Zhao et al. clearly points to the strong involvement of the dynamic (thermal) motion of the oxygen ions: an increase of the oxygen mass by about $10 \%$ drives down $T_{\mathrm{c}}$ by roughly $10 \%$. One of the prime proponents of the importance of Jahn-Teller effects in manganites has been theorist Andrew Millis. His classical model ${ }^{5}$ concentrates on a structural ('Jahn-Teller') transition, rather than on electronic transport and magnetic ordering; as such it does not incorporate the oxygen mass explicitly and therefore does not address the current data.

A simple, and perhaps simplistic, guess at the isotope shift of $T_{\mathrm{c}}$ might go as follows: neglecting lattice fluctuations and their coupling to the electronic and magnetic properties, the transition tempera- ture is $T_{\mathrm{c}, \mathrm{O}}$; accounting for the fluctuations will depress $T_{\mathrm{c}}$ below $T_{\mathrm{c}, \mathrm{O}}$; a heavier mass allows smaller fluctuations and will result in less depression of $T_{\mathrm{c}}$. Thus, $T_{\mathrm{c}}$ increases with mass. Zhao et al., however, report that the heavier mass $(M)$ decreases $T_{c}$, so that the isotope shift $\alpha=\operatorname{dlog}\left(T_{\mathrm{c}}\right) / \operatorname{dlog} M$ is negative.

The negative shift, opposing the simple effect of fluctuations, lends credence to Zhao and colleagues' suggestion that the primary effect is of polaronic origin. The Jahn-Teller polaron is a charge carrier accompanied by a local Jahn-Teller distortion; that is, the charge and local distortion effectively move through the material as a single entity (a polaron). It has a kinetic energy $W_{\text {eff }}$ that is oxygen-mass dependent, and decreases with increasing mass. Because $T_{\mathrm{c}}$ scales with $W_{\text {eff }}$, the isotope shift is negative. Zhao et al. also point out that the variation of the polaronic bandwidth with $\mathrm{La} / \mathrm{Ca}$ ionic radius can account for the trend to larger isotope shifts for smaller mean ion size on the La-Ca sublattice.

Does this news point to the mechanism of CMR? Not directly, it seems, although it shows that dynamical behaviour is an essential component of the behaviour near $T_{\mathrm{c}}$. An important question, still open, is this: how can a system with such strong polaronic character near $T_{\mathrm{c}}$, in which carrier motion is impeded by its strong coupling to atomic motions, become such a good conductor at low temperature? Values of resistivity as low as $10 \mu \Omega \mathrm{cm}$ have been reported, and this amount of resistivity can be accounted for simply by charge scattering from the randomly distributed $\mathrm{La}^{3+}$ and $\mathrm{Ca}^{2+}$ ions. The excellent conduction is consistent with simple band metal behaviour, but there is no theory to account for how the strong electronlattice coupling would 'evaporate' at low temperature.

These new isotope shift results allow one to put a new slant on our picture of the CMR materials. Near $T_{\mathrm{c}}$, there is a huge increase in resistivity resulting from the heavier oxygen isotope - that is, these are also 'colossal isotoporesistance' materials. It is intriguing to note that the effects of the isotopic mass increase (a lowering of $T_{\mathrm{c}}$ ) can, at least approximately, be offset by the application of a magnetic field of the proper strength, which will drive $T_{\mathrm{c}}$ back up to its original value. The essential questions about CMR materials are unanswered, but some of the issues are being clarified.

Warren Pickett is at the Naval Research Laboratory, 4555 Overlook Avenue, Washington, DC 20375-5000, USA.

1. Kim, D. J. Phys. Rev. Lett. 47, 1213-1216 (1981)

2. Zhao, G.-m., Conder, K., Keller, H. \& Müller, K. A. Nature 381, 676-678 (1996).

3. Asamitsu, A. et al. Nature 373, 407-409 (1995).

4. Tomioka, Y. et al. Phys. Rev. B53, R1689-R1692 (1996).

5. Millis, A. J. Phys. Rev. B53, 8434-8441 (1996)

\section{How desire dies}

MANY a woman complains about the short attention span of her lover. After orgasm he suddenly ceases to reiterate his love and passion; instead, he just turns over and goes to sleep. The speed of this orgasmic mental transformation demands explanation. The depletion of a sexual hormone would take far too long. The one thing that changes mood this fast is the sudden injection of a powerful drug. Heroin, nicotine and the adrenaline of our panic syndrome work in seconds. Clearly the male system is flooded at orgasm with some equally fast-acting passionkilling metabolite. Not until this has slowly cleared away can sexual interest return. Women have a different sexual chemistry, and can climax repeatedly.

And this, says Daedalus, explains the failure of the centuries-old search for a true male aphrodisiac. There is no such thing; there is only an anti-aphrodisiac, whose absence from the body allows sexual interest to develop. The brain must have receptors which turn off sexual desire when the compound binds to them.

Some pharmaceuticals can also deaden sexual desire, as an unwelcome side effect. They include antidepressants such as thioridazine and fluoxetine, betablockers such as atenolol, and even bromide - the British Army has been suspected of lacing soldiers' tea with bromide to dampen their sexual urges. Presumably these compounds or their metabolites bind more or less strongly to the brain's anti-aphrodisiac receptors.

So DREADCO's biochemists are seeking the male anti-aphrodisiac in donated blood samples. Daedalus suspects that it builds up in the male system with age. The blood of sexually obsessed teenagers should be almost free of it; that of sexually retired elderly gentlemen should be its richest natural source. It should soar dramatically in a volunteer seducer just after a conquest. Compounds whose concentration varies in the right way will be checked for molecular similarity to the sex-sabotaging drugs. Once identified, the antiaphrodisiac will be synthesized for sale.

DREADCO's 'Passion-Killer' will abolish sexual desire in seconds. As a natural male product, it will be free of the feminizing side effects of previous hormonal anti-aphrodisiacs. Any man in compromising circumstances - a sexual criminal anxious not to re-offend, a diplomat cornered by some Mata Hari, or a politician striving to uphold family values - can simply 'pop' a pill and be released from temptation. Many a sexually reluctant woman will welcome it too, as a crafty additive to her husband's evening meal, or the candle-lit dinner of some unalluring lover. David Jones 